\title{
Cosmology-marginalized approaches in Bayesian model comparison: The neutrino mass as a case study
}

\author{
S. Gariazzo ${ }^{*}$ and O. Mena ${ }^{\dagger}$ \\ Instituto de Física Corpuscular, CSIC-Universitat de València, Paterna, Valencia E-46980, Spain
}

(Received 17 December 2018; published 24 January 2019)

\begin{abstract}
We propose here a novel method which singles out the a priori unavoidable dependence on the underlying cosmological model when extracting parameter constraints, providing robust limits which only depend on the considered dataset. Interestingly, when dealing with several possible cosmologies and interpreting the Bayesian preference in terms of the Gaussian statistical evidence, the preferred model is much less favored than when only two cases are compared. As a working example, we apply our approach to the cosmological neutrino mass bounds, which play a fundamental role not only in establishing the contribution of relic neutrinos to the dark matter of the Universe but also in the planning of future experimental searches of the neutrino character and of the neutrino mass ordering.
\end{abstract}

DOI: 10.1103/PhysRevD.99.021301

\section{INTRODUCTION}

Bayesian parameter inference has been extremely successful in cosmology and astroparticle physics in the past two decades. This statistical technique is more powerful and adequate than traditional tools when dealing with large and complex datasets and with the impossibility to obtain different realizations of the object to study, our Universe. In addition, the Bayesian probability theory has also been extensively exploited for model comparison purposes, offering not only the possibility of predicting but also of optimizing the most adequate theoretical frameworks to fit the cosmological observations; see, e.g., [1]. However, despite the major accomplishments achieved by Bayesian parameter inference, both the role of parametrizations/ priors and the possibility of different fiducial cosmologies (or models) may lead to divergent predictions. The former have caused controversial arguments in the literature, particularly when extracting cosmological bounds on neutrino masses and their ordering [2-7].

In this paper, we shall focus on the potential that Bayesian model comparison techniques offer for computing model-marginalized cosmological parameter limits, avoiding the biases due to the fiducial cosmology. We propose here a simple method to compute such solid and robust model-marginalized constraints.

In order to demonstrate the validity and robustness of this method, we shall illustrate a particular case and consider the sum of the neutrino mass $\Sigma m_{\nu}$ (see Refs. [8-10] for its key signatures on cosmology). Focusing exclusively on bounds from cosmic microwave background (CMB) measurements, the final analyses from the Planck satellite set a

\footnotetext{
gariazzo@ific.uv.es

omena@ific.uv.es
}

95\% C.L. limit of $\Sigma m_{\nu}<0.24 \mathrm{eV}$ [11] after considering CMB temperature, polarization, and lensing at all scales. Late-time observations of the large-scale structure in the Universe by means of the baryon acoustic oscillation (BAO) method sharpen the limit above, as they help enormously in removing the degeneracies present in CMB data at the background level. Once BAO information is combined with Planck measurements, the limit is tightened to $\Sigma m_{\nu}<0.12 \mathrm{eV}$ at $95 \%$ C.L. [11], or even down to $\Sigma m_{\nu}<0.11 \mathrm{eV}$ when also considering supernovae Ia luminosity distances. One obvious question is how reliable and stable are the cosmological neutrino mass limits quoted above?

Even if not relying on the combination of potentially inconsistent datasets for which the neutrino mass bounds become tighter [12], all of the aforementioned limits are based on the most economical $\Lambda \mathrm{CDM}$ scenario, which also leads to the tightest constraints on the neutrino mass. Surely, the bounds on $\Sigma m_{\nu}$ change when (a) new physics is added in the neutrino sector (for instance, changing the effective number of relativistic degrees of freedom, $N_{\text {eff }}$ [14-22] or adding nonstandard interactions [23-36]), (b) new physics appears in the early- or late-time accelerating periods in the Universe [22,37-56] and/or, in general, (c) phenomenologically extended scenarios are considered [57]. While one would naively expect that the neutrino mass limits within these more general cosmologies will always be relaxed, this has been shown not to be the case for physical dark energy models [58,59], for which the neutrino mass bounds get tighter than those obtained in the $\Lambda \mathrm{CDM}$ framework. It is therefore clear that one can artificially tune the cosmological neutrino mass limits in an optimistic or in a pessimistic manner.

These $a$ priori harmless uncertainties translate into very serious dilemmas for neutrino particle physics searches. 
The near and far future neutrinoless double beta decay road map provides a very important example. It seems therefore mandatory to build a method to extract model-independent cosmological neutrino mass bounds. It is among our major goals to apply our novel model-marginalized method to $\Sigma m_{\nu}$ when studying a number of possible cosmological scenarios, i.e., the minimal $\Lambda \mathrm{CDM}$ universe with massive neutrinos and its extensions. Adopting Planck 2015 data [60], the tightest bound we obtain within a $\Lambda$ CDM universe is $\Sigma m_{\nu}<$ $0.23 \mathrm{eV}$ at $95 \%$ C.L., which relaxes to $\Sigma m_{\nu}<0.35 \mathrm{eV}$ when the uncertainty on the cosmological model is taken into account using our model-marginalization method.

At the same time, we can use Bayesian tools in order to compare the models we are studying and obtain which one is preferred by the data. Noticeably, even if the best scenario is strongly favored over its competitors when comparing pairs of models with a Bayes factor analysis, its global statistical evidence falls abruptly when all the models are considered simultaneously, making this preferred model less likely. In the scenarios explored here, this will imply that the weak-to-moderate Bayesian preference for the minimal $\Lambda \mathrm{CDM}+\Sigma m_{\nu}$ model, which arises when it is compared with each of its extensions individually, will not correspond to a global $1 \sigma$ level strength when considering the entire ensemble of extended scenarios.

\section{BAYESIAN STATISTICS}

The Bayes theorem, which represents the foundation of Bayesian statistics, reads

$$
p\left(\theta \mid d, \mathcal{M}_{i}\right)=\frac{\pi\left(\theta \mid \mathcal{M}_{i}\right) \mathcal{L}(\theta)}{Z_{i}},
$$

where $\pi\left(\theta \mid \mathcal{M}_{i}\right)$ and $p\left(\theta \mid d, \mathcal{M}_{i}\right)$ are the prior and posterior probabilities for the parameters $\theta$ within a model $\mathcal{M}_{i}, \mathcal{L}(\theta)$ is the likelihood as a function of the parameters $\theta$, given the data $d$ and the model $\mathcal{M}_{i}$, and $Z_{i}=\int d \theta \pi\left(\theta \mid \mathcal{M}_{i}\right) \mathcal{L}(\theta)$ is the Bayesian evidence of $\mathcal{M}_{i}$ [1]. The Bayes theorem can also be written in a slightly different form to obtain the model posterior probability [61]:

$$
p_{i} \equiv p\left(\mathcal{M}_{i} \mid d\right)=\frac{\pi_{i} Z_{i}}{\sum_{j} \pi_{j} Z_{j}},
$$

where $\pi_{i} \equiv \pi\left(\mathcal{M}_{i}\right)$ refers to the model prior probability. In the Bayesian model comparison framework, the so-called Bayes factor provides a measure of whether the data have increased or decreased the odds of model $\mathcal{M}_{i}$ relative to a second model $\mathcal{M}_{j}$ :

$$
B_{i j}=Z_{i} / Z_{j} \text {. }
$$

The Bayes factor enters the definition of the posterior probability ratio between two models, which indicates how much one of the two is preferred over the other, after using the information provided by the data:

$$
\frac{p_{i}}{p_{j}}=B_{i j} \frac{\pi_{i}}{\pi_{j}} .
$$

If the two models are equivalent according to our initial knowledge, i.e., the model priors are the same, the final preference driven by the data is determined by the Bayes factor. In terms of posterior odds, the preference for the favored model is $B_{i j}: 1$, if $\mathcal{M}_{i}$ is preferred over $\mathcal{M}_{j}$. Adopting the commonly exploited Jeffreys scale [62], the strength of the posterior odds can be ranked as inconclusive $\left(\left|\ln B_{i j}\right|<1\right)$, weak $\left(1<\left|\ln B_{i j}\right|<2.5\right)$, moderate $\left(2.5<\left|\ln B_{i j}\right|<5\right)$, or strong $\left(\left|\ln B_{i j}\right|>5\right)$. Very importantly, this arises from the fact that when comparing two mutually exclusive models, the mentioned ranks correspond roughly to what is usually indicated as a $\lesssim 1 \sigma$ (inconclusive) to $\gtrsim 3 \sigma$ (strong) level when considering a Gaussian variable.

Using Eq. (2) and selecting one among the available models labeled $\mathcal{M}_{0}$ without loss of generality, one can write, provided all priors are identical for all models:

$$
p_{0}=\frac{Z_{0}}{\sum_{i} Z_{i}}=\left(\sum_{i}^{N} B_{i 0}\right)^{-1},
$$

where we have used the definition of the Bayes factor. Notice that the posterior probability of the selected model $\mathcal{M}_{0}$ depends on the Bayes factors with respect to all the possible models. For each data combination, we will choose $\mathcal{M}_{0}$ to be the preferred model. In practice, this is the one that has more influence on the model-marginalized posterior. Since the model $\mathcal{M}_{0}$ is the preferred one, we will always have $B_{i 0}=Z_{i} / Z_{0}<1$ (or $\ln B_{i 0}<0$ ) for $i \neq 0$.

Assuming that (i) more than two models are possible and (ii) all the models have the same prior probabilities, then Eq. (5) implies that the posterior probability of the preferred model is smaller than what the single Bayes factors would suggest in a one-to-one comparison. For example, if $N=8$ and all the Bayes factors are $\left|\ln B_{i 0}\right| \simeq 5$ for $i \neq 0$, thus, indicating apparently strong results according to the usually adopted Jeffreys scale, the posterior probability of $\mathcal{M}_{0}$ is $p_{0} \simeq 0.955$, which would indicate a mild $2 \sigma$ significance for a Gaussian measure. In the same way, having $N=7$ and $\left|\ln B_{i 0}\right| \simeq 2.5$ for $i \neq 0$, which usually indicates a weak preference, would give $p_{0} \simeq 0.67$, which would correspond to a less than $1 \sigma$ preference for $\mathcal{M}_{0}$.

The tools of model comparison also allow us to compute a model-marginalized posterior distribution for the parameter $\theta$, taking into account the posterior probability of each model $\mathcal{M}_{i}$ resulting from the data $d[1]$ :

$$
p(\theta \mid d)=\sum_{i}^{N} p\left(\theta \mid d, \mathcal{M}_{i}\right) p_{i},
$$

where the posterior probabilities of $\theta$ within each model $\mathcal{M}_{i}$ are weighted according to the model posterior probabilities $p_{i}$. These can be written using Eq. (2) to obtain the fundamental formula 


$$
p(\theta \mid d)=\sum_{i}^{N} p\left(\theta \mid d, \mathcal{M}_{i}\right) Z_{i} / \sum_{j}^{N} Z_{j} .
$$

This is the expression that we will use to obtain modelmarginalized limits in the following, under the assumption that all the models have the same priors.

Some final comments are due. To obtain the most robust model-marginalized estimate, one should in principle consider the largest number of possible models. In the cosmological context, these should include the $\Lambda \mathrm{CDM}$ and all its possible extensions, plus scenarios with any possible modified gravity paradigm and their extensions: This is clearly computationally impossible. From an Occam's razor perspective, however, the models with an unnecessarily large number of parameters will be generally penalized by the Bayesian evidence calculation [63] so that their final weight in Eq. (7) will be negligible, while most of the contribution will be given by the most economical models that better fit the data. While our method allows us to marginalize over the freedom related to different models or additional parameters, since it is based on the comparison of Bayesian evidences obtained in the different models, it still has a residual dependence on the shape and the width of the adopted priors.

\section{COSMOLOGICAL DATA ANALYSES}

The data we shall exploit to derive model-marginalized constraints from cosmological observations include measurements of the CMB angular power spectrum and of the $\mathrm{BAO}$ signature in the matter power spectrum. Awaiting the final release from the Planck Collaboration, we use here their 2015 data release $[64,65]$. We consider two possibilities: (a) both temperature and low- $\ell$ polarization $(\mathrm{CMB})$ or (b) temperature and polarization at all multipoles $(\mathrm{CMB}+\mathrm{pol})$. In both cases, we also include the Planck CMB lensing determination (lens) [66]. BAO geometrical information from the SDSS BOSS DR11 [67], the 6DF [68], and the SDSS DR7 MGS [69] surveys complements the datasets used in our numerical analyses. We are aware that this combination may not provide the strongest cosmological constraints; however, it is not our main goal here to outperform the current cosmological constraints but to exemplify the novel model-marginalized approach here proposed. After the Planck final public release, our method will be applied to an extended set of cases with respect to those considered here.

In our numerical calculations, we use the Boltzmann solver CAMB [70] together with COSMOMC [71], with POLYCHORD [61,72] (version 1.9) as the algorithm devoted to extract the Bayesian evidence.

In our demonstrative analysis, we restrict our set of models to the simplest $\Lambda \mathrm{CDM}$ model with freely varying neutrino masses and some of its one-parameter extensions. In particular, we consider the $\Lambda \mathrm{CDM}+\Sigma m_{\nu}, \Lambda \mathrm{CDM}+$ $\Sigma m_{\nu}+A_{\text {lens }}, \Lambda \mathrm{CDM}+\Sigma m_{\nu}+N_{\text {eff }}$, and $\Lambda \mathrm{CDM}+\Sigma m_{\nu}+w$ models, as discussed more in detail in the next paragraphs. In the numerical calculations, all the parameters that are shared among the different models are sampled adopting the same linear priors as in the default POLYCHORD settings, except for the sum of the neutrino masses which is varied in the range $[0.06,5] \mathrm{eV}$. For the additional parameters, we adopt linear priors in the following ranges: $A_{\text {lens }}$ varies in $[0,5], N_{\text {eff }}$ in $[1,5]$, and $w$ in $[-3,0]$.

\section{RESULTS: THE NEUTRINO MASS AS A CASE STUDY}

Table I summarizes the results from our novel method applied to a particular physics case that is usually constrained by cosmological observations: the sum of the neutrino masses $\Sigma m_{\nu}$. As aforementioned, a robust modelmarginalized limit on $\Sigma m_{\nu}$ is absolutely required, as it is crucial for a number of issues. In particular, it is a very important input when deciding the experimental strategy for neutrino character (Dirac vs Majorana) searches. We show such a model-marginalized limit in the second-to-last

TABLE I. 95\% C.L. upper limits on $\Sigma m_{\nu}$ and Bayes factors in the different cosmological scenarios. Results are obtained either adopting Planck 2015 CMB temperature, low- $\ell$ polarization, and lensing data [64-66] plus BAO measurements [67-69] (second and third columns) or the same data combination plus high-multipole CMB polarization measurements from the Planck 2015 data release (fourth and fifth columns). The different rows depict the bounds in different extensions of the $\Lambda \mathrm{CDM}$ model, while the last two rows illustrate, respectively, the modelmarginalized $95 \%$ C.L. limit obtained via Eq. (7) and the posterior probability of the example model $\mathcal{M}_{0}$ (the preferred one that is always the $\Lambda \mathrm{CDM}+\Sigma m_{\nu}$ scenario); see Eq. (5).

\begin{tabular}{|c|c|c|c|c|}
\hline \multirow[b]{2}{*}{ Model } & \multicolumn{2}{|c|}{$\mathrm{CMB}+$ lens $+\mathrm{BAO}$} & \multicolumn{2}{|c|}{$\mathrm{CMB}+$ pol + lens $+\mathrm{BAO}$} \\
\hline & $\ln B_{i 0}$ & $\Sigma m_{\nu}(\mathrm{eV})$ & $\ln B_{i 0}$ & $\Sigma m_{\nu}(\mathrm{eV})$ \\
\hline Base $=\Lambda \mathrm{CDM}+\Sigma m_{\nu}$ & 0.0 & $<0.28$ & 0.0 & $<0.23$ \\
\hline Base $+A_{\text {lens }}$ & -2.6 & $<0.38$ & -2.4 & $<0.29$ \\
\hline Base $+N_{\text {eff }}$ & -1.5 & $<0.37$ & -2.3 & $<0.25$ \\
\hline Base $+w$ & -1.4 & $<0.42$ & -0.1 & $<0.42$ \\
\hline Marginalized & $\cdots$ & $<0.33$ & $\cdots$ & $<0.35$ \\
\hline$p_{0}$ & 0.65 & & 0.48 & \\
\hline
\end{tabular}


row of Table I for the two data combinations considered here.

In order to compute the model-marginalized result we consider, together with the simplest $\Lambda \mathrm{CDM}$ model with freely varying neutrino masses, some cosmological scenarios which are usually explored in the literature; see, e.g., Planck Collaboration 2015 data analyses [65]. These models contain extra parameters which are either partially or significantly degenerate with the neutrino mass. For instance, $\Sigma m_{\nu}$ has a correlation, among others, with the phenomenological parameter $A_{\text {lens }}$, which rescales the lensing amplitude in the $\mathrm{CMB}$ spectra. Since current CMB constraints on the neutrino mass are mostly due to the reduction in the lensing potential induced by a larger neutrino mass, there is a degeneracy between $\Sigma m_{\nu}$ and $A_{\text {lens: }}$ : A value $A_{\text {lens }}<1(>1)$ would allow for a lower (higher) value of $\Sigma m_{\nu}$. Notice from the results depicted in Table I that the neutrino mass bounds, in the absence of high-multipole polarization, are worsened when the $A_{\text {lens }}$ parameter is allowed to vary. When going from a $\Lambda \mathrm{CDM}$ to a $\Lambda \mathrm{CDM}+A_{\text {lens }}$ scenario, the $95 \%$ C.L. limit changes from $\Sigma m_{\nu}<0.28 \mathrm{eV}$ to $\Sigma m_{\nu}<0.38 \mathrm{eV}$ [73]. Another parameter potentially degenerate with $\Sigma m_{\nu}$ is the number of relativistic degrees of freedom $N_{\text {eff }}$, albeit the latest Planck analyses have shown that data are able to disentangle between the different physical effects induced by $\Sigma m_{\nu}$ and $N_{\text {eff }}$ [11] on temperature and polarization anisotropies. While the $95 \%$ C.L. limit without high- $\ell$ polarization is $\Sigma m_{\nu}<0.37 \mathrm{eV}$, information from high multipoles brings the neutrino mass constraint extremely close to the bound obtained within the $\Lambda$ CDM model [74]. Finally, a freely varying constant dark energy equation of state $w$ can also affect the bounds on $\Sigma m_{\nu}$. If $w$ is allowed to vary, the matter energy density can take very high values, compensating for the suppression induced in large-scale structure due to an increased value of $\Sigma m_{\nu}$, and, therefore, these two parameters will be correlated in a significant way. As a result, the limit is relaxed to $\Sigma m_{\nu}<0.42 \mathrm{eV}$ at $95 \%$ C.L., both without and with high-multipole polarization data; see Table I.

From all the limits above and using the Bayes factors also listed in Table I, by means of Eq. (7) it is possible to obtain the marginalized limits on $\Sigma m_{\nu}$ shown in the second-to-last row of Table I. Notice that the 95\% C.L. upper limits obtained within the most economical $\Lambda \mathrm{CDM}$ picture are significantly relaxed (they are increased up to 50\%) when considering extended scenarios. For a visual comparison of the onedimensional posterior probabilities of $\Sigma m_{\nu}$ in the various models considered here and of the model-marginalized one, we provide Figs. 1 and 2, where we also show the sampled prior distribution [75]. Notice that the method following Eq. (7) allows a proper weighing of the information from each model, building a robust estimate for the neutrino mass that can be used as an input in neutrino particle physics. The possible applications of the method, however, are significantly wider than what is explored here.

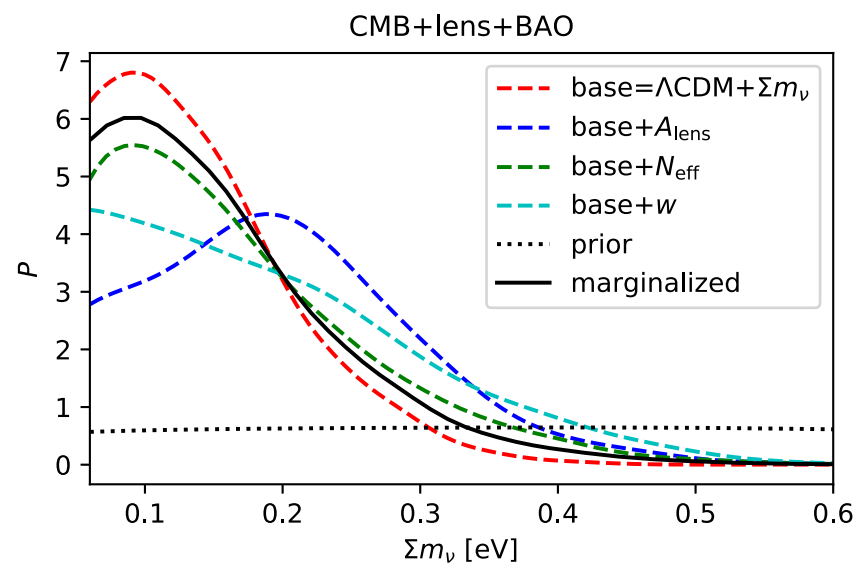

FIG. 1. One-dimensional posterior probabilities for $\Sigma m_{\nu}$ for different cosmological models arising from Planck 2015 CMB temperature, low- $\ell$ polarization, and lensing data [64-66] plus BAO measurements [67-69]. We also depict the modelmarginalized bound obtained using Eq. (7) and the prior sampled on $\Sigma m_{\nu}$; see text for details.

The last row of Table I shows the posterior probabilities $p_{0}$ for the example model $\mathcal{M}_{0}$ computed from Eq. (5). $\mathcal{M}_{0}$ is chosen to be the preferred one by each of the two data combinations, and it turns out to be the minimal $\Lambda \mathrm{CDM}$ scenario with free neutrino masses in both cases. The posterior probability, which depends on the Bayesian evidence of various models, is shown in the second and fourth columns for the two possible data combinations. Here, one should clarify an important aspect of Bayesian model comparison. While the Bayes factors with respect to the extended models, if considered separately, indicate a weak-to-moderate [76] Bayesian preference for the $\Lambda \mathrm{CDM}$ model accordingly to the Jeffreys scale [62], and therefore individually corresponding to a $1.1-2.7 \sigma$ probability (in Gaussian terms) in favor of the $\Lambda$ CDM framework, it is clear from Eq. (5) that such naive expectations are no

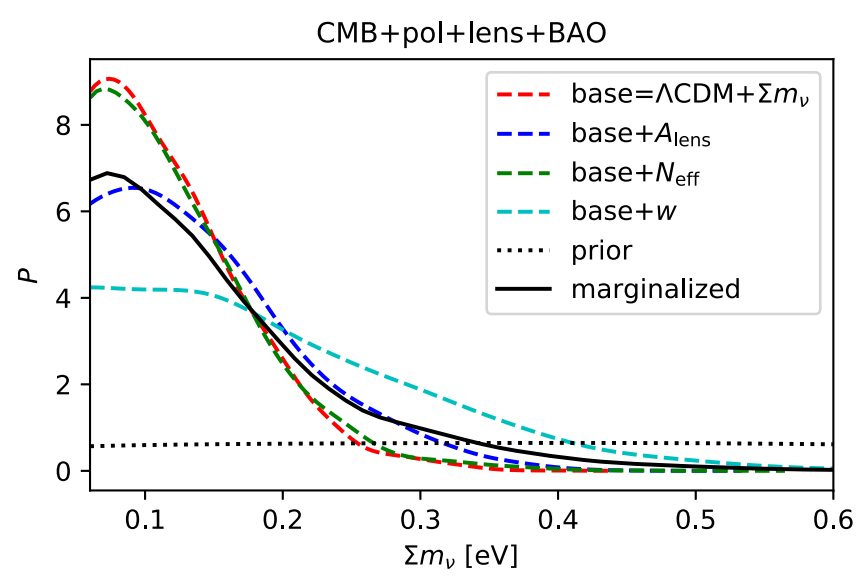

FIG. 2. As Fig. 1 but including also CMB high-multipole polarization measurements from the Planck 2015 data release $[64,65]$. 
longer true when more than one model is accessible. The values of the posterior probabilities for the example $\Lambda \mathrm{CDM}$ model never reach the $1 \sigma$ level strength in terms of a Gaussian variable. Based on these results, therefore, it is possible to say that the $\Lambda \mathrm{CDM}$ model, despite being more likely than its extensions, is not strongly preferred by the data. This is a crucial result of our analyses, with strong implications in many other early Universe fundamental physics searches, as, e.g., in the case of the inflationary landscape, where many models arise and are usually ranked by means of Bayesian comparison techniques; see, e.g., Ref. [77].

\section{DISCUSSION}

Bayesian model comparison provides a robust machinery to compute model-marginalized limits. We have proposed a method which allows us to minimize the uncertainty related to multiple model choices on the determination of parameter constraints. We have applied our novel method to the neutrino mass case, exploiting current publicly available cosmological data. We have shown that the limits on the neutrino masses can significantly change when one realizes that present measurements are not able to unambiguously tell us the cosmological model that nature has chosen. The statistical Gaussian preference for the favored model, indeed, always becomes inconclusive when there are a number of other possible models, even if equally disfavored by observations. An updated and extended analysis using the proposed method will come after the release of Planck 2018 likelihoods.

\section{ACKNOWLEDGMENTS}

We thank J. Lesgourgues for his presentation at Neutrino 2018, which inspired the idea of this paper, and M. Lattanzi and S. Pastor for useful comments on the draft and enlightening discussions. This work is supported by the Spanish Grants No. FPA2015-68783REDT, No. FPA2017-90566-REDC (Red Consolider MultiDark), No. FPA2017-85216-P, No. FPA201785985-P, and No. SEV-2014-0398 (AEI/FEDER, UE, MINECO), and Grants No. PROMETEOII/2014/050, No. PROMETEOII/2018/165, and No. GV2016-142 (Generalitat Valenciana). S. G. receives support from the European Union's Horizon 2020 research and innovation program under the Marie Skłodowska-Curie individual Grant Agreement No. 796941. O. M. is also supported by the European Union's Horizon 2020 research and innovation program under the Marie Skłodowska-Curie Grant Agreements No. 690575 and No. 674896.
[1] R. Trotta, Contemp. Phys. 49, 71 (2008).

[2] F. Simpson, R. Jimenez, C. Peña-Garay, and L. Verde, J. Cosmol. Astropart. Phys. 06 (2017) 029.

[3] T. Schwetz et al., arXiv:1703.04585.

[4] S. Gariazzo, M. Archidiacono, P. F. de Salas, O. Mena, C. A. Ternes, and M. Tórtola, J. Cosmol. Astropart. Phys. 03 (2018) 011.

[5] A. J. Long, M. Raveri, W. Hu, and S. Dodelson, Phys. Rev. D 97, 043510 (2018).

[6] A. F. Heavens and E. Sellentin, J. Cosmol. Astropart. Phys. 04 (2018) 047.

[7] W. Handley and M. Millea, arXiv:1804.08143.

[8] J. Lesgourgues, G. Mangano, G. Miele, and S. Pastor, Neutrino Cosmology (Cambridge University Press, Cambridge, England, 2013).

[9] M. Lattanzi and M. Gerbino, Front. Phys. 5, 70 (2018).

[10] P. De Salas, S. Gariazzo, O. Mena, C. Ternes, and M. Tórtola, Front. Astron. Space Sci. 5, 36 (2018).

[11] N. Aghanim et al. (Planck Collaboration), arXiv:1807.06209.

[12] As an example, adding the prior on the Hubble constant provided by Ref. [13], one would get $\Sigma m_{\nu}<0.0970 \mathrm{eV}$ at 95\% C.L. [11].

[13] A. G. Riess, S. Casertano, W. Yuan, L. Macri, J. Anderson, J. W. MacKenty, J. B. Bowers, K. I. Clubb, A. V. Filippenko, D. O. Jones, and B. E. Tucker, Astrophys. J. 855, 136 (2018).
[14] J. Hamann, S. Hannestad, G. Raffelt, and Y. Y. Wong, J. Cosmol. Astropart. Phys. 08 (2007) 021.

[15] J. Hamann, S. Hannestad, G. G. Raffelt, I. Tamborra, and Y. Y. Wong, Phys. Rev. Lett. 105, 181301 (2010).

[16] E. Giusarma, M. Corsi, M. Archidiacono, R. de Putter, A. Melchiorri, O. Mena, and S. Pandolfi, Phys. Rev. D 83, 115023 (2011).

[17] J. Hamann, S. Hannestad, G. G. Raffelt, and Y. Y. Y. Wong, J. Cosmol. Astropart. Phys. 09 (2011) 034.

[18] E. Giusarma, R. De Putter, and O. Mena, Phys. Rev. D 87, 043515 (2013).

[19] S. Riemer-Sorensen, D. Parkinson, T. M. Davis, and C. Blake, Astrophys. J. 763, 89 (2013).

[20] M. Archidiacono, E. Giusarma, S. Hannestad, and O. Mena, Adv. High Energy Phys. 2013, 191047 (2013).

[21] E. Di Valentino, A. Melchiorri, and O. Mena, J. Cosmol. Astropart. Phys. 11 (2013) 018.

[22] M. Archidiacono, E. Giusarma, A. Melchiorri, and O. Mena, Phys. Rev. D 87, 103519 (2013).

[23] J. F. Beacom, N. F. Bell, and S. Dodelson, Phys. Rev. Lett. 93, 121302 (2004).

[24] N. F. Bell, E. Pierpaoli, and K. Sigurdson, Phys. Rev. D 73, 063523 (2006).

[25] S. Hannestad, J. Cosmol. Astropart. Phys. 02 (2005) 011.

[26] R. Fardon, A. E. Nelson, and N. Weiner, J. Cosmol. Astropart. Phys. 10 (2004) 005. 
[27] N. Afshordi, M. Zaldarriaga, and K. Kohri, Phys. Rev. D 72, 065024 (2005).

[28] A. W. Brookfield, C. van de Bruck, D. Mota, and D. Tocchini-Valentini, Phys. Rev. D 73, 083515 (2006).

[29] A. Brookfield, C. van de Bruck, D. Mota, and D. TocchiniValentini, Phys. Rev. Lett. 96, 061301 (2006).

[30] O. E. Bjaelde, A. W Brookfield, C. van de Bruck, S. Hannestad, D. F. Mota, L. Schrempp, and D. TocchiniValentini, J. Cosmol. Astropart. Phys. 01 (2008) 026.

[31] D. Mota, V. Pettorino, G. Robbers, and C. Wetterich, Phys. Lett. B 663, 160 (2008).

[32] K. Ichiki and Y.-Y. Keum, J. High Energy Phys. 06 (2008) 058.

[33] C. Boehm, M. J. Dolan, and C. McCabe, J. Cosmol. Astropart. Phys. 12 (2012) 027.

[34] M. Archidiacono and S. Hannestad, J. Cosmol. Astropart. Phys. 07 (2014) 046.

[35] G. Dvali and L. Funcke, Phys. Rev. D 93, 113002 (2016).

[36] E. Di Valentino, C. B. e. hm, E. Hivon, and F. R. Bouchet, Phys. Rev. D 97, 043513 (2018).

[37] J. Hamann, S. Hannestad, M. S. Sloth, and Y. Y. Wong, Phys. Rev. D 75, 023522 (2007).

[38] S. Joudaki, Phys. Rev. D 87, 083523 (2013).

[39] R. de Putter, E. V. Linder, and A. Mishra, Phys. Rev. D 89, 103502 (2014).

[40] E. Di Valentino, S. Gariazzo, M. Gerbino, E. Giusarma, and O. Mena, Phys. Rev. D 93, 083523 (2016).

[41] N. Canac, G. Aslanyan, K. N. Abazajian, R. Easther, and L. C. Price, J. Cosmol. Astropart. Phys. 09 (2016) 022.

[42] M. Gerbino, K. Freese, S. Vagnozzi, M. Lattanzi, O. Mena, E. Giusarma, and S. Ho, Phys. Rev. D 95, 043512 (2017).

[43] D. Huterer and E. V. Linder, Phys. Rev. D 75, 023519 (2007).

[44] M. Baldi, F. Villaescusa-Navarro, M. Viel, E. Puchwein, V. Springel, and L. Moscardini, Mon. Not. R. Astron. Soc. 440, 75 (2014).

[45] B. Hu, M. Raveri, A. Silvestri, and N. Frusciante, Phys. Rev. D 91, 063524 (2015).

[46] J. Shim, J. Lee, and M. Baldi, arXiv:1404.3639.

[47] A. Barreira, B. Li, C. Baugh, and S. Pascoli, Phys. Rev. D 90, 023528 (2014).

[48] N. Bellomo, E. Bellini, B. Hu, R. Jimenez, C. Peña-Garay, and L. Verde, J. Cosmol. Astropart. Phys. 02 (2017) 043.

[49] S. Peirone, N. Frusciante, B. Hu, M. Raveri, and A. Silvestri, Phys. Rev. D 97, 063518 (2018).

[50] J. Renk, M. Zumalacárregui, F. Montanari, and A. Barreira, J. Cosmol. Astropart. Phys. 10 (2017) 020.

[51] Y. Dirian, Phys. Rev. D 96, 083513 (2017).

[52] M. B. Gavela, D. Hernandez, L. Lopez Honorez, O. Mena, and S. Rigolin, J. Cosmol. Astropart. Phys. 07 (2009) 034; 05 (2010) E01.

[53] B. A. Reid, L. Verde, R. Jimenez, and O. Mena, J. Cosmol. Astropart. Phys. 01 (2010) 003.

[54] L. Lopez Honorez and O. Mena, AIP Conf. Proc. 1241, 1016 (2010).

[55] G. La Vacca, S. Bonometto, and L. Colombo, New Astron. 14, 435 (2009).
[56] R.-Y. Guo, J.-F. Zhang, and X. Zhang, Chin. Phys. C 42, 095103 (2018).

[57] E. Di Valentino, A. Melchiorri, and J. Silk, Phys. Rev. D 92, 121302 (2015).

[58] S. Vagnozzi, S. Dhawan, M. Gerbino, K. Freese, A. Goobar, and O. Mena, Phys. Rev. D 98, 083501 (2018).

[59] S. R. Choudhury and S. Choubey, J. Cosmol. Astropart. Phys. 09 (2018) 017.

[60] Although the final data analyses have already been presented by the Planck Collaboration, the data and likelihood codes are not public at the time of writing.

[61] W. J. Handley, M. P. Hobson, and A. N. Lasenby, Mon. Not. R. Astron. Soc. 453, 4384 (2015).

[62] H. Jeffreys, Theory of Probability, The International Series of Monographs on Physics (Clarendon Press, Oxford, 1961).

[63] This is true as long as the additional parameters are constrained by the data, as no penalty applies to unconstrained parameters. The selection of models considered in the analysis should take into account equal priors only for reasonable extensions of the simplest model.

[64] R. Adam et al. (Planck Collaboration), Astron. Astrophys. 594, A1 (2016).

[65] P. A. R. Ade et al. (Planck Collaboration), Astron. Astrophys. 594, A13 (2016).

[66] P. A. R. Ade et al. (Planck Collaboration), Astron. Astrophys. 594, A15 (2016).

[67] L. Anderson et al. (BOSS Collaboration), Mon. Not. R. Astron. Soc. 441, 24 (2014).

[68] F. Beutler, C. Blake, M. Colless, D. H. Jones, L. StaveleySmith, L. Campbell, Q. Parker, W. Saunders, and F. Watson, Mon. Not. R. Astron. Soc. 416, 3017 (2011).

[69] A. J. Ross, L. Samushia, C. Howlett, W. J. Percival, A. Burden, and M. Manera, Mon. Not. R. Astron. Soc. 449, 835 (2015).

[70] A. Lewis, A. Challinor, and A. Lasenby, Astrophys. J. 538, 473 (2000).

[71] A. Lewis and S. Bridle, Phys. Rev. D 66, 103511 (2002).

[72] W. J. Handley, M. P. Hobson, and A. N. Lasenby, Mon. Not. R. Astron. Soc. 450, L61 (2015).

[73] As a comparison, the Planck Collaboration 2015 data analyses quote $\Sigma m_{\nu}<0.25 \mathrm{eV}$ and $\Sigma m_{\nu}<0.41 \mathrm{eV}$ for these two cases, clearly stating the very good consistency of the results here obtained.

[74] The 2015 Planck results provide a 95\% C.L. upper limit of $\Sigma m_{\nu}<0.32 \mathrm{eV}\left(\Sigma m_{\nu}<0.22 \mathrm{eV}\right)$ without (with) the addition of high- $\ell$ polarization measurements in the $\Lambda \mathrm{CDM}+N_{\text {eff }}$ model.

[75] While, in principle, the prior sampled by CosmoMC can be nontrivial, the distribution turns out to be flat in the parameter region of interest here, making our results strongly independent on the prior choice.

[76] One exception is represented by the $\Lambda \mathrm{CDM}+w$ model when full polarization data are included, which is basically equivalent to the $\Lambda \mathrm{CDM}$ model. All the Bayes factors we obtain from our numerical calculation, however, carry an uncertainty of $\sim 0.3$.

[77] P. A. R. Ade et al. (Planck Collaboration), Astron. Astrophys. 594, A20 (2016). 